\title{
Comparisons of the risk of myopericarditis between COVID-19 patients and individuals receiving COVID-19 vaccines: a population-based study
}

Oscar Hou In Chou MSc ${ }^{1,2}$, Jiandong Zhou $\mathrm{PhD}^{3}$, Teddy Tai Loy Lee ${ }^{4}$, Thompson Kot MBChB ${ }^{5}$, Sharen Lee ${ }^{2}$, Abraham Ka Chung Wai MBChB FRCEM FRCP ${ }^{4}$, Wing Tak Wong PhD ${ }^{6}$, Qingpeng Zhang $\mathrm{PhD}^{3}$, Shuk Han Cheng PhD ${ }^{7}$, Tong Liu MD PhD FESC ${ }^{8}$, Vassilios S Vassiliou MBBS PhD FRCP FESC FACC \# ${ }^{9}$, Bernard Man Yung Cheung MB BChir PhD FRCP $\#^{1}$, Gary Tse MD PhD FRCP FFPH \# ${ }^{1,8,10}$

${ }^{1}$ Division of Clinical Pharmacology, Department of Medicine, University of Hong Kong, Hong Kong, China

${ }^{2}$ Epidemiology Research Unit, Cardiovascular Analytics Group, Hong Kong, China-UK Collaboration

${ }^{3}$ School of Data Science, City University of Hong Kong, Hong Kong, China

${ }^{4}$ Emergency Medicine Unit, University of Hong Kong, Hong Kong, China

${ }^{5}$ Department of Anaesthesia, Princess Margaret Hospital, Hong Kong, China

${ }^{6}$ School of Life Sciences, Chinese University of Hong Kong, Hong Kong, China

7 Department of Infectious Diseases and Public Health, City University of Hong Kong, China

${ }^{8}$ Tianjin Key Laboratory of Ionic-Molecular Function of Cardiovascular Disease, Department of Cardiology, Tianjin Institute of Cardiology, Second Hospital of Tianjin Medical University, Tianjin 300211, China

${ }^{9}$ Department of Cardiology, Norwich Medical School, University of East Anglia, Norfolk and Norwich University Hospital, Floor 2, Bob Champion Building, James Watson Road, Norwich, NR4 7UQ, UK

${ }^{10}$ Kent and Medway Medical School, Canterbury, Kent, UK

Word count: 904 words

\# Correspondence to:

Vassilios Vassiliou MBBS PhD FRCP FESC FACC

Professor of Cardiac Medicine, Department of Cardiology, Norwich Medical School,

University of East Anglia, Norfolk and Norwich University Hospital, Floor 2, Bob Champion Building, James Watson Road, Norwich, NR4 7UQ, UK

Email: v.vassiliou@uea.ac.uk

Bernard Man Yung Cheung, MB BChir PhD FRCP

Clinical Professor, Department of Medicine, Li Ka Shing Faculty of Medicine, University of Hong Kong, China

Honorary Consultant, Department of Medicine, Queen Mary Hospital, Hong Kong, China

Email: mycheung@hku.hk

Gary Tse MD PhD FRCP FFPH

Reader in Public Health, Kent and Medway Medical School, Canterbury, Kent, UK 
Professor, Tianjin Key Laboratory of Ionic-Molecular Function of Cardiovascular Disease, Department of Cardiology, Tianjin Institute of Cardiology, Second Hospital of Tianjin Medical University, Tianjin 300211, China

Principal Investigator, Epidemiology Research Unit, Cardiovascular Analytics Group, Hong Kong, China-UK Collaboration

Email: garytse86@gmail.com

\title{
Words: 997
}

\begin{abstract}
Background: Both COVID-19 infection and COVID-19 vaccines have been associated with the development of myopericarditis. The objective of this study is to 1) analyze the rates of myopericarditis after COVID-19 infection and COVID-19 vaccination in Hong Kong and 2) compare to the background rates, and 3) compare the rates of myopericarditis after COVID-19 vaccination to those reported in other countries.
\end{abstract}

Methods: This was a population-based cohort study from Hong Kong, China. Patients with positive RT-PCR test for COVID-19 between $1^{\text {st }}$ January 2020 and $30^{\text {th }}$ June 2021 or individuals who received COVID-19 vaccination until $31^{\text {st }}$ August were included. The main exposures were COVID19 positivity or COVID-19 vaccination. The primary outcome was myopericarditis.

Results: This study included 11441 COVID-19 patients from Hong Kong, of whom four suffered from myopericarditis (rate per million: 350; 95\% confidence interval [CI]: 140-900). The rate was higher than the pre-COVID-19 background rate in 2020 (rate per million: 61, 95\% CI: 55-67) with a rate ratio of 5.73 (95\% CI: 2.23-14.73. Compared to background rates, the rate of myopericarditis among vaccinated subjects in Hong Kong was substantially lower (rate per million: 8.6; 95\% CI: 6.411.6) with a rate ratio of 0.14 (95\% CI: $0.10-0.19)$. The rates of myocarditis after vaccination in Hong Kong are comparable to those vaccinated in the United States, Israel, and the United Kingdom. 
Conclusions: COVID-19 infection is associated with a higher rate of myopericarditis whereas COVID-19 vaccination is associated with a lower rate of myopericarditis compared to the background.

\section{Introduction}

Since the beginning of the COVID-19 pandemic, cases of myocarditis and pericarditis related to the infection have been widely described. ${ }^{1,2}$ However, recent studies have also reported possible associations between COVID-19 vaccines and the risk of myo-pericardial inflammation, raising concerns about vaccination uptake especially in teenagers. In this study, we conducted a populationbased study using data from Hong Kong, China to determine the rates of myopericarditis after COVID-19 infection and COVID-19 vaccination, comparing them to background rates and addressing the relative risk of vaccine vs COVID-19 myopericarditis.

\section{Methods}

This population-based retrospective cohort study was approved by the Institutional Review Board of the University of Hong Kong/Hospital Authority Hong Kong West Cluster (UW 20-250). The need for informed consent was waived by the Ethics Committee owing to its observational retrospective nature. Patients who tested positive for COVID-19 by real-time polymerase chain reaction (RT-PCR) at any of the Hong Kong public hospitals or outpatient clinics between $1^{\text {st }}$ January 2020 to $30^{\text {th }}$ June 2021 were included. The data were obtained from the local electronic healthcare database, Clinical Data Analysis and Reporting System, as reported previously ${ }^{3}$. The primary outcome was myopericarditis. The vaccination data of other countries were extracted using keywords labelled "Myocarditis" and "Pericarditis" upon searching PubMed and the official reports of Hong Kong and the United Kingdom. The data in Hong Kong and the United Kingdom was up to $31^{\text {st }}$ August 2021 and $29^{\text {th }}$ September 2021, respectively. 
The rates of myocarditis and pericarditis after COVID-19 infection were calculated by dividing the number of patients by the number of RT-PCR positive COVID-19 patients. The number of doses was defined as the sum of the number of people who received the first and second doses. The hybrid Wilson/Brown method was used to calculate $95 \%$ confidence intervals for proportions. The analysis was conducted using PRISM (Version: 9.0.0).

\section{Results}

A total of 11441 COVID-19 patients from Hong Kong were included, of which four suffered from myopericarditis (rate per million: 350; 95\% CI: 140-900). This rate was higher than the prepandemic background rate in 2020 (rate per million: 61, 95\% CI: 55-67) with a rate ratio of 5.73 (95\% CI: 2.23-14.73; Table 1). Compared to background rates, the rate of myopericarditis among vaccinated subjects in Hong Kong was substantially lower (rate per million: 8.6; 95\% CI: 6.4-11.6) with a rate ratio of 0.14 (95\% CI: $0.10-0.19)$. The rates of myomyocarditis after vaccination in Hong Kong are comparable to those vaccinated in the United States, Israel, and the United Kingdom (Figure 1).

\section{Discussion}

The main finding from our study is that COVID-19 patients have a higher rate of myopericarditis compared to the pre-COVID-19 era. With COVID-19 vaccination, the rate of myopericarditis was significantly lower than the background rates and the rate in COVID-19 positive patients.

Our team recently developed a predictive model to identify COVID-19 patients at risk of severe disease ${ }^{4}$. Using an updated dataset, we found that $0.035 \%$ of the COVID-19 infected patients developed myopericarditis. This is significantly higher than the baseline, reflecting the association 
between COVID-19 infection and myopericarditis. Previously, among 718,365 patients with COVID-19, approximately $6.5 \%$ developed new-onset myopericarditis. ${ }^{5}$ This higher rate might be an overestimate since most of the patients presented with mild COVID-19 symptoms may not get admitted and diagnosed, thus, not registered with the electronic medical records. Furthermore, our cases represented population-based data locally in Hong Kong, which has practised meticulous contact tracing since the pandemic; the number of total COVID-19 patients in our cohort would, therefore, likely reflect most of the infected patients in the community.

The link between COVID-19 vaccination and myopericarditis has been raised in Israel in May 2021. The results demonstrated that COVID-19 vaccinations are associated with a lower myopericarditis rate than the COVID-19 infected patients. This indicated that the COVID-19 vaccination might protect the vaccinated people from the myocardial injury caused by SARS-CoV-2 infection. ${ }^{6}$ The differences in the outcome suggested that the myopericarditis upon vaccination is unlikely due to the mimicry between the spike protein. ${ }^{7}$ While the rate of myopericarditis is even lower than the background in our data; this does not rule out the causation between the COVID-19 vaccine and myopericarditis. Indeed, the majority of the myopericarditis developed after the second dose of the vaccine among the younger male $\left(<19\right.$ years old). ${ }^{2}$ While the rate of myopericarditis following COVID-19 infection is relatively low; further studies are needed to characterise the mechanism of myopericarditis after COVID-19 vaccination. Nonetheless, with the current increasing numbers in COVID-19 globally, it would appear that ever person will come in contact with SARS$\mathrm{CoV}-2$, therefore the overall risk of myopericarditis will be much higher at a population level without vaccination.

\section{Strengths and limitations}


This study has several strengths. Firstly, COVID-19 cases were identified by RT-PCR testing across the public sector. Therefore, missing cases are likely to be few. Secondly, possible cases of vaccine-related myopericarditis were reviewed by an expert panel, that examined the medical records independently. This adjudication has permitted the accurate classification of cases according to established international guidelines. Nevertheless, had we included possible cases rather than cases definitely linked to vaccinations, the rate ratios of myopericarditis cases in infected patients to cases occurring after COVID-19 vaccination would be even lower, and therefore does not alter our conclusion.

However, several limitations should be noted. Firstly, the cohort included patients recruited from a single region and as such, is unable to account for any geographical heterogeneity that may exist. Secondly, the vaccination data reported in the local Department of Health did not provide the number of myopericarditis after the first and the second dose of vaccination and therefore the results are not stratified. Thirdly, we might have missed COVID-19 myopericarditis in patients who might have died from acute heart failure complications due to myopericarditis, but recorded as heart failure deaths. This however, would have increased the difference between COVID-19 and vaccine related myopericarditis, further strengthening the usefulness of vaccination.

\section{Conclusion}

COVID-19 infection is associated with significantly higher rate of myopericarditis compared to both background rates and vaccine associated myopericarditis. 
medRxiv preprint doi: https://doi.org/10.1101/2021.12.13.21267730; this version posted December 14, 2021. The copyright holder for this preprint (which was not certified by peer review) is the author/funder, who has granted medRxiv a license to display the preprint in perpetuity.

It is made available under a CC-BY 4.0 International license .

\section{References}

1. Witberg G, Barda N, Hoss S, et al. Myocarditis after Covid-19 Vaccination in a Large Health Care Organization. N Engl J Med 2021 2021/10/07. DOI: 10.1056/NEJMoa2110737.

2. Mevorach D, Anis E, Cedar N, et al. Myocarditis after BNT162b2 mRNA Vaccine against Covid-19 in Israel. N Engl J Med 2021 2021/10/07. DOI: 10.1056/NEJMoa2109730.

3. Tse G, Zhou J, Lee $S$, et al. Relationship between angiotensin-converting enzyme inhibitors or angiotensin receptor blockers and COVID-19 incidence or severe disease. J Hypertens 2021; 39: 1717-1724. 2021/07/01. DOI: 10.1097/HJH.0000000000002866.

4. Zhou J, Lee $S$, Wang $X$, et al. Development of a multivariable prediction model for severe COVID-19 disease: a population-based study from Hong Kong. NPJ Digit Med 2021; 4: 66. 2021/04/10. DOI: 10.1038/s41746-021-00433-4.

5. Buckley BJR, Harrison SL, Fazio-Eynullayeva E, et al. Prevalence and clinical outcomes of myocarditis and pericarditis in 718,365 COVID-19 patients. European Journal of Clinical Investigation; n/a: e13679. DOI: https://doi.org/10.1111/eci.13679.

6. Imazio M, Klingel K, Kindermann I, et al. COVID-19 pandemic and troponin: indirect myocardial injury, myocardial inflammation or myocarditis? Heart 2020; 106: 1127-1131. DOI:

10.1136/heartjnl-2020-317186.

7. Bozkurt B, Kamat I and Hotez PJ. Myocarditis With COVID-19 mRNA Vaccines. Circulation 2021; 144: 471-484. DOI: doi:10.1161/CIRCULATIONAHA.121.056135.

8. Drug Office of the Department of Health HKS, China Adverse Drug Reactions (Reporting) https://www.drugoffice.gov.hk/eps/do/en/healthcare providers/adr reporting/index.html (2021, accessed 10 October 2021).

9. Diaz GA, Parsons GT, Gering SK, et al. Myocarditis and Pericarditis After Vaccination for COVID-19. JAMA 2021; 326: 1210-1212. DOI: 10.1001/jama.2021.13443.

10. GOV.UK. Coronavirus vaccine - weekly summary of Yellow Card reporting https://www.gov.uk/government/publications/coronavirus-covid-19-vaccine-adversereactions/coronavirus-vaccine-summary-of-yellow-card-reporting (2021, accessed 10 October 2021). 


\begin{tabular}{|c|c|c|c|c|c|c|}
\hline Outcomes & $\begin{array}{c}\text { Background } \\
\text { (Hong Kong, China) }^{8}\end{array}$ & $\begin{array}{l}\text { Covid-19 infection } \\
\text { (Hong Kong, China) }\end{array}$ & $\begin{array}{l}\text { COVID-19 vaccine } \\
\text { (Hong Kong, China) }^{8}\end{array}$ & $\begin{array}{c}\text { COVID-19 vaccines } \\
\text { (US })^{9}\end{array}$ & $\begin{array}{c}\text { COVID-19 vaccines } \\
\text { (Israel) }^{2}\end{array}$ & $\begin{array}{c}\text { COVID-19 vaccines } \\
(\text { (UK) })^{10}\end{array}$ \\
\hline Diagnosis & Myopericarditis & Myopericarditis & Myopericarditis & Myopericarditis & Myocarditis & Myopericarditis \\
\hline Type of vaccines & NA & NA & $\begin{array}{l}\text { CoronaVac } \\
\text { BNT162b2 }\end{array}$ & $\begin{array}{c}\text { BNT162b2 } \\
\text { mRNA-1273 } \\
\text { Ad26.COV2.S } \\
\end{array}$ & BNT162b2 & $\begin{array}{c}\text { BNT162b2 } \\
\text { mRNA-1273 } \\
\text { ChAdOx1 } \\
\end{array}$ \\
\hline Cases & 416 & 4 & 42 & 37 & 136 & 947 \\
\hline Unit & Persons & Persons & Doses & Doses & Doses & Doses \\
\hline $\begin{array}{c}\text { Persons or doses } \\
\text { received }\end{array}$ & 6819672 & 11441 & 4883721 & 3530507 & 10568331 & 93600000 \\
\hline $\begin{array}{c}\text { Rate per million } \\
\text { persons or doses }(95 \% \\
\mathrm{CI}) \\
\end{array}$ & $\begin{array}{c}61 \\
(55,67)\end{array}$ & $\begin{array}{c}350 \\
(140,900)\end{array}$ & $\begin{array}{c}8.6 \\
(6.4,11.6)\end{array}$ & $\begin{array}{c}10.5 \\
(7.6,14.4)\end{array}$ & $\begin{array}{c}12.9 \\
(10.9,15.2)\end{array}$ & $\begin{array}{c}10.1 \\
9.5,10.8\end{array}$ \\
\hline Rate Ratio (95\% CI) & 1 (baseline) & $5.73(2.23,14.73)$ & $0.14(0.10 .0 .19)$ & $0.17(0.12,0.24)$ & $0.21(0.18,0.25)$ & $0.17(0.16,0.18)$ \\
\hline
\end{tabular}

Table 1. Incidence rate and rate ratio of myocarditis and pericarditis after COVID-19 infection and vaccination. * Cases for $>=12$ years old only. 


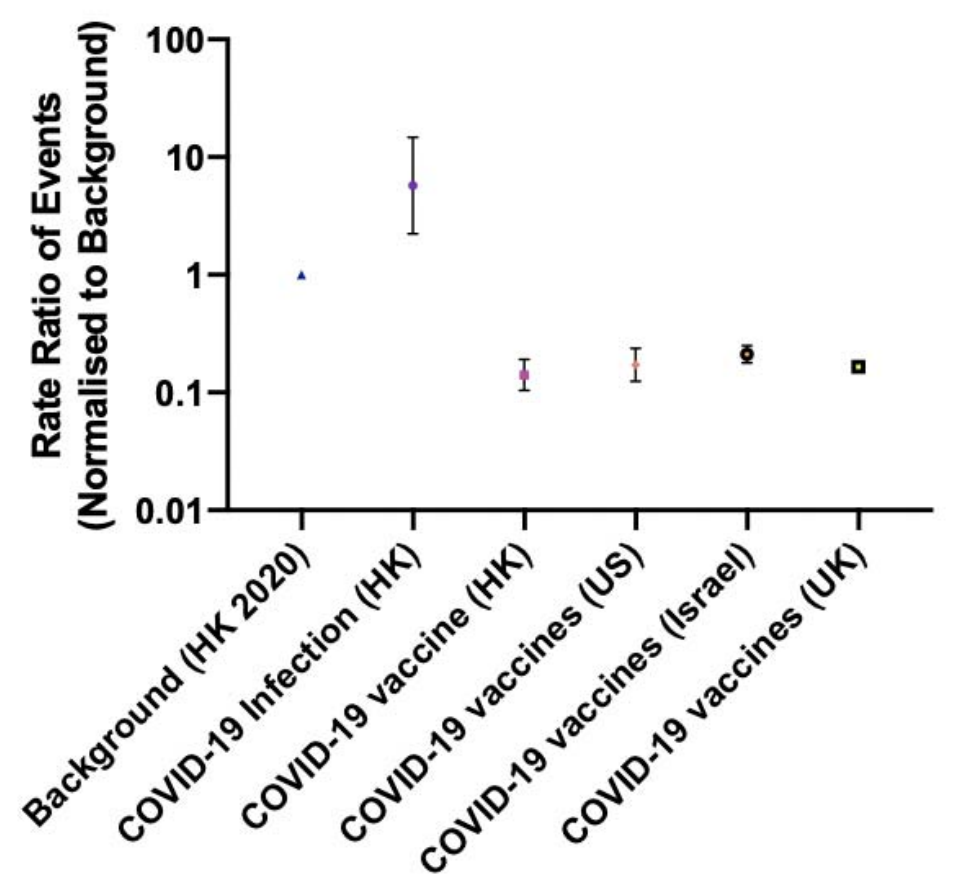

Figure 1. The rate ratio of the events after COVID-19 infection and COVID-19 vaccination. 
medRxiv preprint doi: https://doi.org/10.1101/2021.12.13.21267730; this version posted December 14, 2021. The copyright holder for this preprint (which was not certified by peer review) is the author/funder, who has granted medRxiv a license to display the preprint in perpetuity.

It is made available under a CC-BY 4.0 International license .

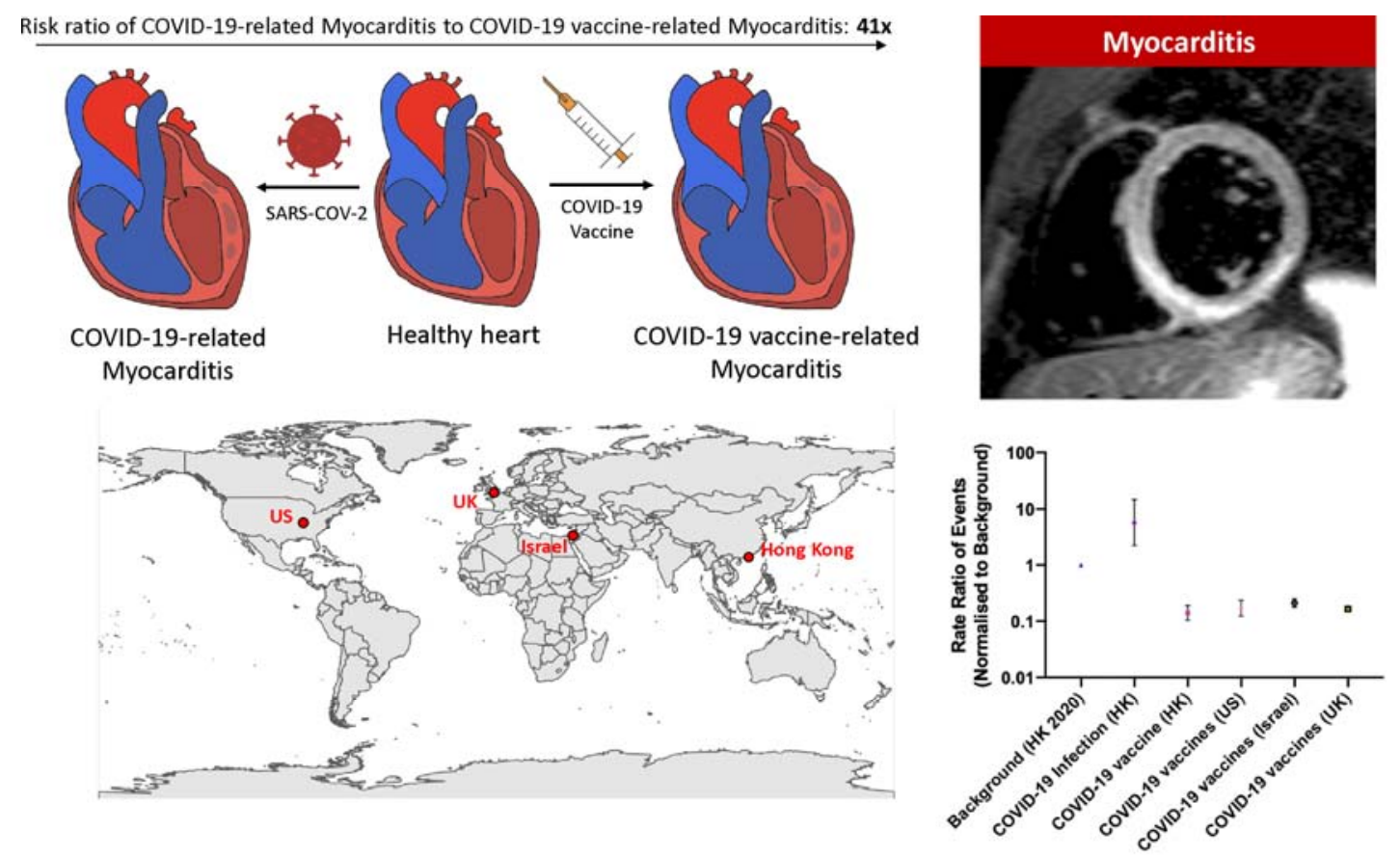

Central illustration 